\title{
Optimal Synthesis and 3D Modeling of a Lifting Mechanism for a Platform with Variable Slope
}

\author{
Eduardo Vázquez-Santacruz, Cuauhtémoc Morales-Cruz, \\ Rogelio de Jesus Portillo-Velez, Mariano Gamboa-Zúñiga \\ Cinvestav-IPN, CGSTIC, Mexico, D.F., Mexico \\ \{efvazquez, cmorales, mgamboaz\}@cinvestav.mx
}

\begin{abstract}
Today, one of the most important research fields is concerned to the Engineering Design Process. It is focused on designing better electro-mechanical systems with a low impact on the environment and a most efficient use of the energy. Currently, the design process can take advantage of many computational-aided tools, which play a crucial role in the modern methods of optimization and reduce the cost of prototyping. On the other hand, a lifting mechanism for a platform that changes its slope in a specified range and moves along this restriction with a distributed load has many applications in different fields. In this work, the synthesis of a lifting mechanism is obtained by proposing and solving a design problem as a nonlinear optimization problem.
\end{abstract}

Keywords: Lifting mechanism, optimal parameterization, kinematic model.

\section{Introduction}

New fields and approaches have emerged to fulfill the design requirements of many disciplines. Medical requirements have been always at the top of demanding fields to satisfy hospitals requirements and more important to improve the patient comfort and quality of the devices that help with his recovery. New mechanical devices must be designed to fulfill successful health-care services. One of the devices that most impact has been the medical hospital bed [10,9]. Several mechanical designs of hospital beds have been proposed to alleviate the very intensive labor and the lack of qualified personnel (nurses and stretcher-bearers), mainly in developing countries. In Ching-Hua [3], a hospital bed with auxiliary functions of lateral positioning and transferring patients is described. Three mechanisms are described which assist in complicated maneuvers of moving patients from hospital beds to the stretcher. Andhare [1] makes a design that attempt is to reduce the amount of assistance required in managing these patients. Special focus is made on the mechanism synthesis stage. Kittipichai [5], proposed an optimization procedure for the structure design of a hospital bed using genetic algorithms. The novelty of the bed structure is that it can support the left and or right leg for patient's leg splint. Some goals of the design are to reduce the mass of the bed structure. In the work of Shih-Wei [7] the 
Mechanism Design and Mechatronic Control of a Multifunctional Test Bed for Bedridden Healthcare is presented. The design considers two beds, one main bed, and one nursing bed with transferring capabilities. A remarkable feature is that the designed bed is built by mechatronic engineers and qualified healthcare personnel simultaneously. Kap-Ho [4] presents the development of an intelligent bed robot system, in particular, a bed equipped with two robot arms and an array of pressure sensors. These sensors are attached to the mattress capable of estimate the pose of the patient. Mohammed [6] present a new design of a Multi-Functional Portable Patient Bed which is used to carry and transfer a patient's body. The most interesting novelty of the approach is that the design is demand-based, i.e. the proposed design of the bed is formulated based on literature survey as well as consult the medical staff. This design approach for the hospital bed goes beyond, and it is based on Latin-American patients, nurse and stretcher-bearers demands. Hospital human resources are the people in daily contact with real situations and needs. For this reason, their feedback is essential to produce a useful hospital bed. This design also plots the basis to consider a functional set of positions demanded by real bed needs. Then, for each required position a mechanism synthesis stage brings a solution for the motion of each required tool. Finally, using tools of mechanical engineering, the complete design can be developed. It is important to mention that the bed construction involves the design and manufacturing of various areas (mechanical, electronic, industrial and graphic design). This integration produces a functional device in combination with an intelligent system [2].

\section{Selection of Lifting Mechanism}

Lifting machines exist in different configurations. They may be movable, stationary or passive, it depends on their application. Also, this machines may have intermittent action or continuous and may be conducted electrically, by a combustion engine, solar energy or by some other power source. Its principal task is to move a load from one initial point to any other of interest, and they must hold the loads along the path and in the final point. Even that must of the machines are required to move its load strictly in a horizontal motion; some others are designed to develop an angular movement while they are transporting the load. In this work, the Lifting Mechanism must be capable of supporting a weight of $150 \mathrm{~kg}$ but must be light and strong for greater mobility. As well as being able to develop a desired angular motion.

\subsection{Morphologie Analysis}

Due to the nature of the design process, there is not a single correct answer for a design problem. Nevertheless, there may be better solutions for the same problem, taking into account the cost-profit. For this reason, it was decided to occupy the Morphology Analysis Methodology [11]. 


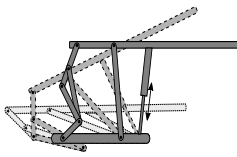

(a)

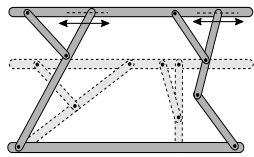

(b)

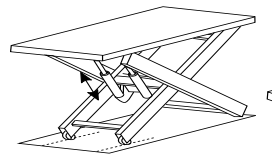

(c)

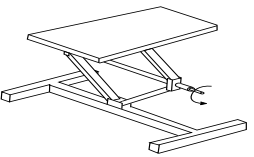

(d)

Fig. 1. Four conceptual designs.

This methodology consists of determining multiple conceptual designs while relying on the requirements and characteristics for a system. Following the method, four options were proposed at the beginning of the process. These mechanisms are shown in Fig. 1: a) Lift Storage Mechanism, b) Lift Bench Mechanism, c) Lift Up Mechanism, and d) Scissor Lift Mechanism. In this four possibilities, the link driver to produce the movement of the system is identified with an arrow next to it. In Table 1 is displayed the list of input requirements for the design of the mechanism. This list considers specific behavior and properties of the desired system to establish which one fulfill more of them.

Table 1. Comparison of the options.

\begin{tabular}{|l|l|l|l|l|}
\hline Characteristics \Evaluated mechanisms & a) & b) & c) & d) \\
\hline Speed & & & & \\
\hline Few elements & & & & \\
\hline Few drivers & & & & \\
\hline No horizontal displacement & & & & \\
\hline Can vary the slope & & & \\
\hline Analysis easiness & & & & \\
\hline Easy manufacturing & & & & \\
\hline Low cost & & & & \\
\hline Stress distribution & & & & \\
\hline
\end{tabular}

\section{$2.2 \quad$ Final Selection}

The four mechanisms were evaluated with the mentioned approach, and the Lift Bench Mechanism ,showed in Figure 1(b), had more advantages over the others. The kinematic model was obtained to describe the detailed behavior of each link. The result was a final proposal configuration for this mechanism.

\section{Description of Mechanism}

\subsection{Description of the Lifting Mechanism}

The lifting device is composed of two subsystems. They can work together to provide not only the vertical motion of the surface but also the ability to change 
the slope of the plane. Figure 2(a) shows a sketch of the entire system with its principal components and actuators to perform the desired behavior. The mechanism one is a four bar mechanism where one of the links is coupled to a linear actuator, marked with the letter $\mathrm{A}$, which changes the position of the slider, thus providing its vertical motion. It is important to mention that one of the links is extended to bond with the mechanism 2. The mechanism two is a five bar mechanism where one of the links is coupled to a linear actuator. In this other case marked with the letter B, this motor changes the position of the slider to produce the vertical motion of this mechanism or to vary the slope of the surface. However, it depends on the relative vertical position of mechanism one to mechanism two. As well as the mechanism one can alter the slope of the platform by changing its relative vertical position to mechanism 2. Figure 1 illustrates that both devices handle the supporting of the whole system. Moreover, working together this two mechanisms can vary the vertical position represented by the variable $h$ in the same illustration. Differential movements from the two actuators produce a slope in the platform of the system measuring the deviation of the normal surface vector from the vertical one. This variable is represented by $\alpha$. The entire system was selected and configured to produce motion in three distinct configurations. The first one consists of moving the platform surface in strictly vertical position. It means that both mechanisms have the same height and work together to achieve the required horizontal configuration along the vertical displacement of the platform. In the other two motion patterns, both mechanisms collaborate to achieve the desired angle variation in one direction and its opposite.

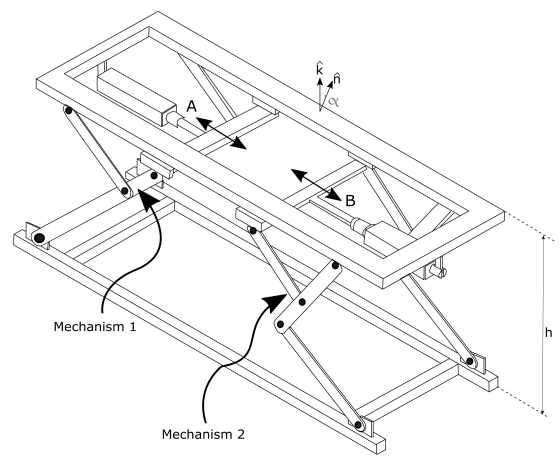

(a)

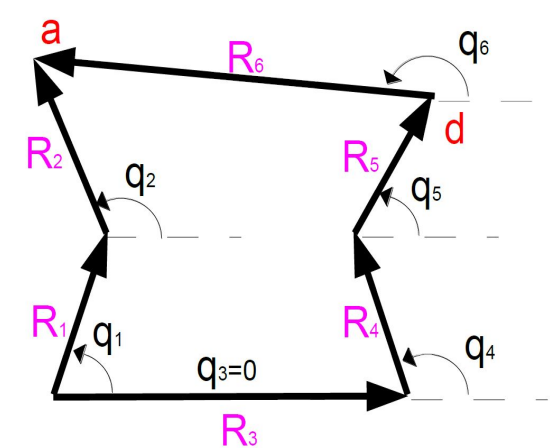

(b)

Fig. 2. Physical and schematic description.

\subsection{Analysis of Position}

A schematic drawing of the lifting machine is shown in Figure 2(b). The entire mechanism can be represented by a six-bar array and it is composed of a reference 
bar $\left(R_{3}\right)$, and five links to make a closed chain. The other links have the labels $R_{1,2,4-6}$. On the other hand, $q_{i} \in \mathbb{R} \forall i=1 \ldots 6$, is the i-th angle between the horizontal axis and the i-th bar with positive counterclockwise direction.

Equation (1) represents the closed chain for the mechanism in Figure 2(b). In this expression each vector is related with each one of the linkages. On the other hand, if vectors are written in polar form [8], (1) can be expressed by equations (2) and (3). Note that when the direction of $\boldsymbol{R}_{\mathbf{6}}$ is zero, the y-coordinate of the point $a$ and the point $d$ are the same. Therefore, the height of the mechanism can be defined by the position of point $a$ or point $d$, when the orientation of the vector $q_{6}$ is zero. The vertical positions $a_{y}$ point and $d_{y}$ are given by equations (4) and (5), respectively. Horizontal positions $a_{x}$ and $d_{x}$ are given by equations (6) and (7) respectively.

$$
\begin{gathered}
\boldsymbol{R}_{\mathbf{1}}+\boldsymbol{R}_{\mathbf{2}}=\boldsymbol{R}_{\mathbf{3}}+\boldsymbol{R}_{\mathbf{4}}+\boldsymbol{R}_{\mathbf{5}}+\boldsymbol{R}_{\mathbf{6}} \\
r_{1} \cos \left(q_{1}\right)+r_{2} \cos \left(q_{2}\right)=r_{3} \cos \left(q_{3}\right)+r_{4} \cos \left(q_{4}\right)+r 5_{c} \cos \left(q_{5}\right)+r_{6} \cos \left(q_{6}\right) \\
r_{1} \sin \left(q_{1}\right)+r_{2} \sin \left(q_{2}\right)=r_{3} \sin \left(q_{3}\right)+r_{4} \sin \left(q_{4}\right)+r_{5} \sin \left(q_{5}\right)+r_{6} \sin \left(q_{6}\right) \\
a_{y}=r_{1} \sin \left(q_{1}\right)+r_{2} \sin \left(q_{2}\right) \\
d_{y}=r_{3} \sin \left(q_{3}\right)+r_{4} \sin \left(q_{4}\right)+r_{5} \sin \left(q_{5}\right) \\
a_{x}=r_{1} \cos \left(q_{1}\right)+r_{2} \cos \left(q_{2}\right) \\
d_{x}=r_{3} \cos \left(q_{3}\right)+r_{4} \sin \left(q_{4}\right)+r_{5} \sin \left(q_{5}\right)
\end{gathered}
$$

\section{Optimization Problem Statement}

The main structure of the system is composed of a six-bar mechanism, and it can be analyzed as a closed kinematic chain. The main objective of this device is to achieve a maximum rise of $90 \mathrm{~cm}$ and a minimum height of $40 \mathrm{~cm}$ from the base of the mechanism. Likewise, a minimum angle of 16 degrees to the Trendelenburg position is required. The six-bar mechanism design problem is proposed as an optimization problem, where the design variables that optimize the performance function proposal are wanted, subject to constraints inherent in the system. The next subsections mention in detail the design variables, performance function and limitations that are part of the optimization problem design. 


\subsection{Design Variables}

The combination of physical parameters is the responsible for the overall system performance. Based on the requirements already defined, the vector $p \in \mathbb{R}^{12}$ described in (8) which synthesizes the system is proposed. Where the variables $r_{1}-r_{6}$ represents the magnitude of the vectors and the variables $q_{1}-q_{6}$ represents the direction of the corresponding vectors. The elements of this vector physically represent the lengths of the links as well as the angles that each link. The chosen parameters create the set of design variables that can be accessed to modify the system performance.

$$
\begin{aligned}
p & =\left[x_{1}, x_{2}, x_{3}, x_{4}, x_{5}, x_{6}, x_{7}, x_{8}, x_{9}, x_{10}, x_{11}, x_{12}\right]^{T} \\
& =\left[r_{1}, r_{2}, r_{3}, r_{4}, r_{5}, r_{6}, q_{1}, q_{2}, q_{3}, q_{4}, q_{5}, q_{6}\right]^{T}
\end{aligned}
$$

\subsection{Objective Function}

$$
\min _{x} I=w_{x}\left(a_{x}-x_{d}\right)^{2}+w_{y}\left(a_{y}-y_{d}\right)^{2}+w_{6}\left(q_{6}-q_{6 d}\right)^{2}
$$

Where $w_{x}, w_{y}$ and $w_{z}$ are weights in the function.

\subsection{Constraints}

The constraints imposed to the optimization problem are the maximum-minimum lengths of the links, and the range of feasible angles for each link. These restrictions are expressed in mathematical form in (10)-(14). The constants parameters are defined in Table 2 .

$$
\begin{gathered}
r_{3} \cos \left(q_{3}\right)+r_{4} \cos \left(q_{4}\right)+r 5_{c} \operatorname{os}\left(q_{5}\right)+r_{6} \cos \left(q_{6}\right)-r_{1} \cos \left(q_{1}\right)-r_{2} \cos \left(q_{2}\right)=0 \\
r_{3} \sin \left(q_{3}\right)+r_{4} \sin \left(q_{4}\right)+r_{5} \sin \left(q_{5}\right)+r_{6} \sin \left(q_{6}\right)-r_{1} \sin \left(q_{1}\right)-r_{2} \sin \left(q_{2}\right)=0 \\
r_{3}-r_{3 d}=0, \quad r_{6}-r_{6 d}=0 \\
q_{3}=0, \quad 6-q_{6 d}=0 \\
r_{1 \min } \leq r_{1} \leq r_{1 \max } \\
r_{2 \min } \leq r_{2} \leq r_{2 \max } \\
r_{4 \min } \leq r_{4} \leq r_{4 \max } \\
r_{5 \min } \leq r_{5} \leq r_{5 \max } \\
q_{1 \min } \leq q_{1} \leq q_{1 \max } \\
q_{2 \min } \leq q_{2} \leq q_{2 \max } \\
q_{4 \min } \leq q_{4} \leq q_{4 \max } \\
r_{5 \min } \leq q_{5} \leq q_{5 \max }
\end{gathered}
$$


Table 2. Constraints

\begin{tabular}{|l|l|l|l|}
\hline$r_{1 \min }=10[\mathrm{~cm}]$ & $r_{4 \min }=10[\mathrm{~cm}]$ & $q_{1 \min }=10^{\circ}$ & $q_{4 \min }=100^{\circ}$ \\
$r_{1 \max }=50[\mathrm{~cm}]$ & $r_{4 \max }=50[\mathrm{~cm}]$ & $q_{1 \max }=80^{\circ}$ & $q_{4 \max }=170^{\circ}$ \\
$r_{2 \min }=10[\mathrm{~cm}]$ & $r_{5 \min }=10[\mathrm{~cm}]$ & $q_{2 \min }=100^{\circ}$ & $q_{5 \min }=10^{\circ}$ \\
$r_{2 \max }=50[\mathrm{~cm}]$ & $r_{5 \max }=50[\mathrm{~cm}]$ & $q_{2 \min }=170^{\circ}$ & $q_{5 \max }=80^{\circ}$ \\
\hline$r_{3 d}=150[\mathrm{~cm}]$ & $r_{6 d}=150[\mathrm{~cm}]$ & $w_{x}=1$ & $w_{y}=1$ \\
\hline$w_{q}=1$ & $x_{d}=0[\mathrm{~cm}]$ & $y_{d}=90[\mathrm{~cm}]$ & $q_{6 d}=180$ \\
\hline
\end{tabular}

\section{Problem Solution and Results}

\subsection{Sequence of the Solving Process}

The optimization toolbox of MatLab was used to solve the nonlinear optimization problem proposed. This tool contains the function fmincon that considers the inequality constraints of the optimization problem statement. Due to the nature of the problem, the active-set section of this function was implemented. This approach computes the Hessian approximation needed to obtain the final solution. Figure 3 shows the flowchart of the solving process. On the other hand, this problem does not have a unique solution. Therefore, the next subsections present two different results that strongly depend on the initial condition given to the solving function.

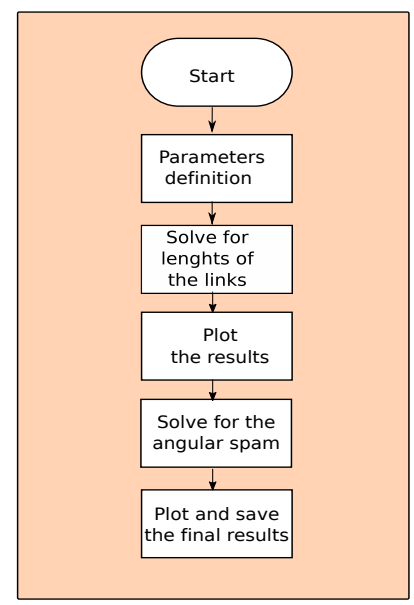

Fig. 3. Optimization flow chart.

\subsection{Case 1:}

The first case contemplates the initial condition is expressed in (15). The vector solution is in (16), and its components represent the solution for the design 
Eduardo Vázquez-Santacruz, Cuauhtémoc Morales-Cruz, Rogelio de Jesus Portillo-Velez, et al.

problem. Figure 4 illustrates a view of the final configuration

$$
\begin{aligned}
X_{01}= & {\left[\begin{array}{llllllllllll}
1 & 0 & 1 & 0 & 0 & 0 & 0 & 0 & 0 & 0 & 0 & 180^{\circ}
\end{array}\right]^{T} } \\
X_{01}^{*}= & {\left[\begin{array}{llllllll}
49.98 & 50 & 150 & 49.99 & 41.95 & 150 & 64.15^{\circ} \\
& 115.82^{\circ} & 0100.81^{\circ} & 77.1^{\circ} & 179.9^{\circ}
\end{array}\right]^{T} }
\end{aligned}
$$

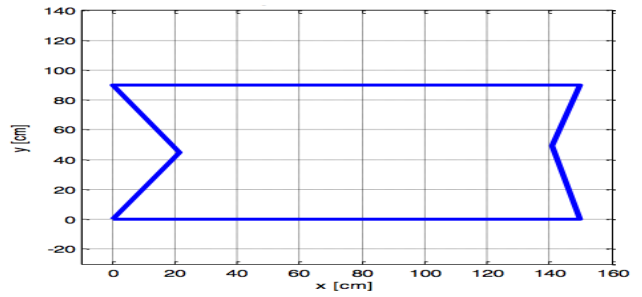

Fig. 4. Configuration for the initial condition 1.

\subsection{Case 2:}

For this second case, it is proposed the initial condition expressed in (17). The vector solution is in (18), and its components represent the solution for the design problem. Figure 5 illustrates a view of this final configuration.

$$
\begin{aligned}
& X_{02}=\left[\begin{array}{lllllllll}
50 & 50 & 150 & 50 & 50 & 150 & 50^{\circ} & 50^{\circ} & 0^{\circ}
\end{array}\right. \\
& \left.\begin{array}{lll}
50^{\circ} & 50^{\circ} & 180^{\circ}
\end{array}\right]^{T} \\
& X_{02}^{*}=\left[\begin{array}{llllll}
46.74 & 46.77 & 150 & 48.89 & 44.04 & 150
\end{array}\right. \\
& 74.23^{\circ} 105.74^{\circ} \quad 0^{\circ} 103.69^{\circ} 74.74^{\circ} \\
& \left.179.98^{\circ}\right]^{T}
\end{aligned}
$$




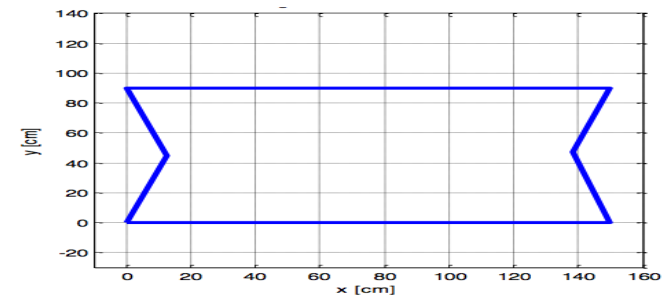

Fig. 5. Configuration mechanism for the initial condition 2.

\subsection{Final Results}

In sum up, Figure 6 represents the maximum and minimum height for each of the mechanism. This two especial configuration are quite important and they were part of the initial requirements. For this specific plot $q_{6}=80^{\circ} \pm 16^{\circ}$.

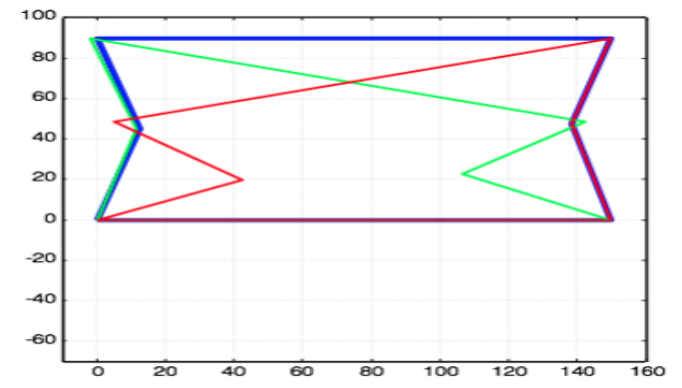

Fig. 6. Complete range for the angular displacement of the platform.

\section{$6 \quad 3 \mathrm{D}$ Modeling}

Modeling a system, not only helps to visualize how the prototype would be physically, but also to prevent bad positions, non-realistic pieces, collisions and many others failures. This problem is quite difficult to detect for the designer engineer without the prototyping. One of the biggest advantages when modeling a system is that any detected failure can be repaired in this process. Most of the time this changes involves many other pieces, which can be re-adapted to the design without too many troubles. It is important to mention that the result of the recursive process used many times, showing clearly the importance of using CAD software. In contrast to the traditional design process, which the prototyping is imperative to detect failures and prevent them in the subsequent models, the new technologies provide the advantages of avoiding the cost of prototyping. It leaves everything in the hands of the design engineer. However the recursive process of designing, proving, detecting failures and redesigning, 
must be kept. The lifting mechanism modeled in this work is composed of two subsystems. Which provide not only the vertical motion of the surface but also the ability to change the slope of the plane. An isometric view of the entire system is shown in the Figure 7(a), as well as the location of the two subsystems, mentioned before.Finally Figure 7(b) shows the distribution of the load with purple arrows. It contemplates the load that the system will be moving with during its operation. However the details will not be covered in this document.

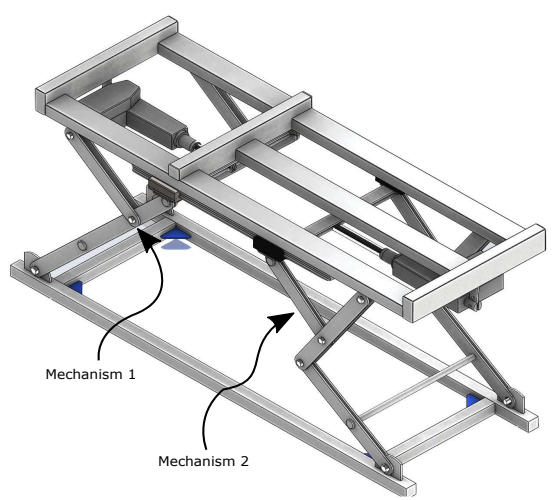

(a)

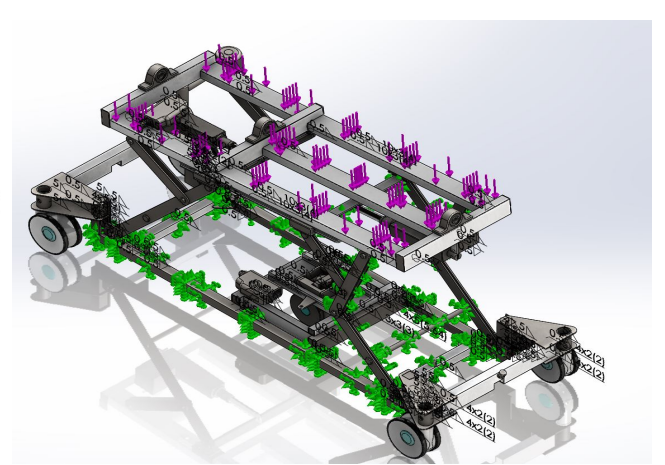

(b)

Fig. 7. Physical and schematic description.

\section{Conclusion}

This paper presented the synthesis of a lifting mechanism as an optimization problem. The analysis of position was carried out and the requirements of the final system were set to guide the process. At the end a complete mechanism was obtained and it is capable of moving according to the restrictions imposed. This work has shown how to take advantage of optimization in order to simplify the design process. Additionally, a 3D model of the lifting mechanism was presented. It has been used 3D CAD software tools for its development. This work contributes, as an example, to the design process, which is currently employed. Despite the traditional process, where most of the time it has to build a prototype, to prove the motion of the system, this work present an alternative methodology with some advantages explained before.

\section{References}

1. Andhare, A., Onkar, A., Padole, P.: Design of bed for bedridden patients: Analysis and synthesis of mechanisms. In: 15th National Conference on Machines and Mechanisms, Chennai, Tamilnadu, India. pp. 1-6 (2011) 
2. Beltrán-Herrera, A., Vázquez-Santacruz, E., Gamboa-Ziga, M.: Real-time classification of lying bodies by HOG descriptors. In: 6th Mexican Conference on Pattern Recognition MCPR. pp. 211-220 (2014)

3. Ching-Hua, W., Ting-Chun, T., Shin-Chieh, H., Wan-Chun, C., Yen-Ming, C., Kun-Tse, T., Chun-Went, Y., Kuo-YiI, C.: Hospital bed with auxiliary functions of lateral positioning and transferring for immobilized patients. In: The 33rd Annual Conference of the IEEE Industrial Electronics Society (IECON). pp. 2991-2995 (2007)

4. Kap-Ho, S., Changmok, O., Tae-Yong, C., Ju-Jang, L.: Bed-type robotic system for the bedridden. In: Proceedings of the 2005 IEEE/ASME International Conference on Advanced Intelligent Mechatronics, Monterey, California, USA. pp. 1170-1175 (2005)

5. Kittipichai, R., Ariyarit, A.: The sizing optimization of hospital bed structure for independently supporting left and or right leg using genetic algorithms. In: International Journal of Modeling and Optimization. vol. 11, pp. 122-188 (2011)

6. Mohammeda, M.N., Khrita, N.G., Abdelgneia, M.A., Abubakera, Es.and Muftaha, A., Omara, M.Z., Salleha, M.S.: A new design of multi-functional portable patient bed. In: Jurnal Teknologi (Sciences \& Engineering). vol. 58, pp. 61-66 (2012)

7. Shih-Wei, P., Feng-Li, L., Li-Chen, F.: Mechanism design and mechatronic control of a multifunctional test bed for bedridden healthcare. In: IEEE/ASME transactions on mechatronics. vol. 15, pp. 234-241 (2010)

8. Uicker, J., Pennock, G., Shigley, J.: Theory of Machines and Mechanisms. McGrawHill series in mechanical engineering, Oxford University Press (2003)

9. Vazquez-Santacruz, E., Gamboa-Zuniga, M.: A diagnosis methodology for assistive technology development. In: 10th International Conference on Electrical Engineering, Computing Science and Automatic Control (CCE). pp. 163-169 (2013)

10. Wang, Y., Butner, S., Darzi, A.: The developing market for medical robotics. Proceedings of the IEEE 9(94), 1763-1771 (2006)

11. Zwicky, F.: Morphologie and Policy Analysis. 16th EURO Conference on Operational Analysis, Brussels (1998) 